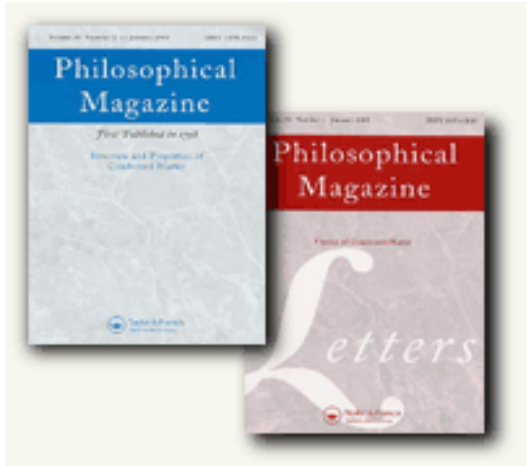

\title{
Contribution of matrix frustration to the free energy of cluster distributions in binary alloys
}

\begin{tabular}{|r|l|}
\hline Journal: & Philosophical Magazine \& Philosophical Magazine Letters \\
\hline Manuscript ID: & TPHM-06-Mar-0044.R1 \\
\hline Journal Selection: & Philosophical Magazine \\
\hline $\begin{array}{r}\text { Date Submitted by the } \\
\text { Author: }\end{array}$ & 07-Apr-2006 \\
\hline Complete List of Authors: & Lepinoux, Joel; LTPCM, INPG \\
\hline Keywords: & $\begin{array}{l}\text { aluminium alloys, annealing, clusters, metallic alloys, modelling, } \\
\text { nucleation, phase decomposition }\end{array}$ \\
\hline Keywords (user supplied): & precipitation, free energy, cluster dynamics \\
\hline
\end{tabular}

\section{s scholarONE" \\ Manuscript Central}




\title{
Contribution of matrix frustration to the free energy of cluster distributions in binary alloys
}

\author{
J. LEPINOUX \\ LTPCM (UMR 5614 CNRS), INPG \\ 38042 St Martin d'Hères, France
}

\begin{abstract}
A generalized version of Frenkel's model of cluster gas is proposed to provide a rigorous description of the contribution of the configuration entropy to the total free energy of cluster distributions in binary alloy. It is shown that the predicted cluster distributions are in excellent agreement with those obtained in kinematical Monte Carlo simulations. The emission and absorption coefficients to be used in cluster dynamics are fully defined: they depend not only of the free energy of clusters but also of the whole cluster distribution. Alternatively, this model can provide accurate values of the nucleation driving force used in classical nucleation theory.
\end{abstract}

Keywords: Free Energy, Precipitation, Cluster Dynamics, Nucleation.

\section{Introduction}

The classical nucleation theory (CNT) and its companion, the cluster dynamics (CD), are still very living subjects which continue to inspire an impressive amount of works. Among the various reasons for this continuous interest, one can find: - the generality of this theory which makes it a tool commonly used in a wide range of fields, each community developing its own culture of the CNT, adapted to its needs; - the increasing need to extend this theory to situations more complex than the model situations examined by pioneers (see [1] as a starting point to appreciate such aspects). In addition there is also a more recent reason: indeed, the continuous development of experimental investigation techniques and computing capabilities allows us to carefully examine some painful approximations of this theory. Kinematical Monte Carlo simulation (KMC) is a privileged tool to help us to better understand how precipitation really takes place in crystals [2]. Contrary to real materials, the properties of the simulated material based on realistic interatomic potentials are perfectly controlled and the role of various quantities can be easily tested. For instance, it is possible to know if monomers are the only species of mobile clusters or not [3]; alternatively it is also possible to design a potential which forbids or allows the mobility of small clusters to study the influence of this mechanism [4]. Therefore, when comparing with analytical approaches it is no longer acceptable to assume any hypothesis for the sake of simplicity or to invoke possible effects to justify observed deviations between models and experiments, for instance. As in various other fields, such mature simulation tool can help us to differentiate the right from the wrong among theories developed in a time where such a tool was not available, yet. A good example of point worth to examine, first in binary systems, is the definition of the well-known absorption and emission coefficients defined in CTN and used in CD calculations. Following the work of Kelton [5], it was recently suggested, in a paper further referred to as paper I [6], to cut the problem into two pieces: interfacial reactions, a purely thermodynamical feature, 
and long range diffusion, whose knowledge is only necessary to predict the precipitation kinetics, i.e. to link the physical time to successive states of the system. Curiously, the first point has been the subject of a controversy for many years while the second point seems so trivial that is seldom discussed, except recently because there is increasing evidence that in many real materials monomers are not the only mobile clusters [2]. Hopefully there are situations where only monomers diffuse, e.g. AlZr and AlSc alloys [3]; such situations are thus perfect case studies to investigate both thermodynamical and kinematical aspects of precipitation.

In paper I, interfacial exchange rates were obtained through an atomic scale analysis of the probabilities to capture and release a solute atom at the interface of a cluster. The ratio of these coefficients was found similar to the ratio of the absorption and emission coefficients in $\mathrm{CD}$, i.e. equal to $\exp \left(-\left(\Delta F_{n+1}-\Delta F_{n}\right) / k_{B} T\right)$ multiplied by a concentration of monomers $\left(k_{B}\right.$ is the Boltzmann constant, $T$ is the temperature and $\Delta F_{n}=\left(F_{n}-n F_{1}\right)$ is the difference of free energy between a cluster of size $n$ and that of $n$ monomers). Of course, in the former case this concentration of monomers is local, while in the latter case it is taken in its global sense. Although these two concentrations are strictly equivalent only at equilibrium, this difference only deals with the kinetics, through the different schemes to solve the master equation (i.e. one or two pairs of coefficients). The main information to retain from this comparison between microscopic and macroscopic analysis is that the thermodynamics is the same in both descriptions, as expected. This confirms, one more time, that the expression of the ratio of these coefficients does not depend on whether the considered clusters are sub- or supercritical. This also means that the most classical way to find this ratio, i.e. by considering the notion of equilibrium concentrations of clusters, is fundamentally correct, despite that the predicted equilibrium concentrations of clusters lose their physical meaning beyond the critical size. In order to avoid this controversial feature, an alternative general demonstration was proposed by Slesov and Schmelzer [7] leading, of course, to the same result. More recently, the link between CNT and CD was examined in a general way by Martin [8].

In fact, the most important is not how the expression of this ratio is obtained, all approaches leading to the same result, but how this ratio is actually calculated. Macroscopic thermodynamics has been suspected for a long time to be unsuitable to describe the free energy of small clusters, classically approximated by the capillary approximation like large clusters. This concern gave rise to original works about 20 years ago to find alternative calculation methods based on statistical physics [9]. Note that alternatives to the capillary approximation have been proposed also for liquids [10] and gases [11]. Using the same kind of method than Perrini et al. [9], various features of clusters, including their free energy, were recently computed for clusters of cubic (or $\mathrm{L}_{12}$ ) structure, taking advantage of current computing capabilities (paper I). In particular, it was shown that the usual constant interface energy is a well suited approximation only beyond a cluster size of about 35 solute atoms (for this structure) and only in a given range of temperature; this threshold was found to increase with temperature. For cluster sizes smaller than this threshold the free energy is more complex to describe, i.e. three parameters are necessary, or if one prefers, the interface energy depends on cluster size. And for very small clusters (less than 10 solute atoms) it is preferable to use the exact polynomial solution (cf. paper I) because it cannot be efficiently fitted with a simple law. Specific difficulties were found to describe the free energy at low or high temperature, see paper I for details. Therefore, these results explain why CTN and CD can be successful in a given range of temperature and solute concentration and fail out of this range. Nevertheless, despite these recent progresses, comparisons between cluster distributions obtained by $\mathrm{CD}$ with those provided by KMC simulations revealed important deviations at high temperature and solute concentrations. As long as only cluster distributions are compared, and not the 
evolutions versus time of average quantities, like the average cluster size for instance, only the thermodynamical description of the alloy is involved, i.e. it should not depend (or only very weakly) on the description of the long distance diffusion process which controls the kinetics. As the accuracy of the calculation of the free energy of clusters is fairly good (of the order of $0.1 \%$ at high temperature) this deviation has another origin which appears when one comes back to the expression of the total free energy of a distribution of clusters. The neglected contribution is the configurational entropy of the so-called cluster gas.

The main goal of the present paper is to examine this contribution. In other words, paper I deals with the arrangement of solute atoms within clusters (the main contribution to the total free energy) while the present work deals with the arrangement of clusters within the system. This question can be envisaged following various points of view, e.g. as a correction to bring to the absorption and emission coefficients to be used in $\mathrm{CD}$, or more generally as an efficient way to provide a reference solution for binary alloys when atomic interactions can be truncated at a distance equal to the lattice parameter $a$ (or the solute atoms sublattice in the case of ordered clusters). The strategy adopted here is based on the notion of cluster gas as known from Frenkel's pioneering work [12]. It will be shown that when associated with the previously mentionned calculations of cluster free energy, this concept can provide a rigorous way to describe the free energy of any distribution of clusters in a binary alloy without the various approximations peculiar to classical methods like Cluster Variational Method (CVM) [13], Low Temperature Expansions (LTE) [14-15] or simpler thermodynamical models like regular or ideal solutions.

Starting from the notion of cluster gas, the derivation scheme leading to the expression of cluster concentrations at equilibrium is first recalled (section 2). Then it is shown step by step how the "frustration" of the matrix can be fully included: this frustration arises from the space forbidden to a given cluster by other clusters (section 3). Section 4 shows how this contribution has to be accounted for in the so-called absorption and emission coefficients in $\mathrm{CD}$. Then the predicted distributions of clusters are compared with those observed in Kinematical Monte Carlo (KMC) simulations for AlZr alloys and finally the link with the Classical Theory of Nucleation (CNT) is examined.

All KMC data reported in this paper were provided by courtesy from E. Clouet; most results and details about the simulation procedure can be found in [3, 16-17] and forthcoming papers.

\section{Notions of equilibrium concentrations and cluster gas}

\subsection{Equilibrium concentrations}

In undersaturated solutions, i.e. of concentration $C_{0}<C_{e q}$, where $C_{e q}$ is the limit of solubility, a stable (Boltzmann) distribution of clusters can develop: it is the distribution which minimizes the total free energy of the system. In such a distribution, the concentration of each class of clusters of size $n$ (where $n$ is the number of solute atoms in the cluster) takes its so-called equilibrium value $\tilde{C}_{n}$ defined by:

$$
\tilde{C}_{n}=\exp \left(-\frac{\Delta G_{n}}{k_{B} T}\right)
$$


in the CNT framework where $\Delta G_{n}$ is the formation free energy of clusters of size $n$. Equivalently, in the cluster gas formalism the same quantity is defined by:

$$
\tilde{C}_{n}=\left[\tilde{C}_{1}\right]^{n} \exp \left(-\frac{\Delta F_{n}}{k_{B} T}\right)
$$

where $\tilde{C}_{1}$ is the equilibrium concentration of monomers.

Figure 1 shows a typical example of cluster distribution for an undersaturated solution $\left(C_{0}=0.75 C_{e q}\right)$. This distribution was obtained using a classical algorithm of cluster dynamics (for recent works see $[17,18]$ ) which strictly speaking is not a true free energy minimization algorithm but it succeeds to reproduce values given by Equation (2) with a relative accuracy which can reach $10^{-10}$.

In a supersaturated solution, such a stable distribution of clusters cannot establish because of the emergence of a critical size $n^{*}$ : instead, the long time behaviour is characterized by the growth of overcritical clusters while subcritical clusters dissolve. Nevertheless, according to the CNT, a "quasi steady-state" is expected, at least for low supersaturated alloys, such that the cluster concentrations still take their equilibrium values for $n<n^{*}$ although the concentration decreases faster than expected according to Equation (2) while approaching $n^{*}$. This deviation was studied in details and extended to non-steady states by Kashchiev [19]; for steady-states, with the free energy of clusters approximated with the capillary approximation it was shown that this deviation equals to $50 \%$ at $n=n^{*}$.

These predictions are in good agreement with cluster distributions obtained in CD for low supersaturated solutions. Figure 2 shows such an example obtained for an alloy of low solubility limit and $C_{0}=5 C_{e q}$; the ratio $1 / 2$ between simulated and predicted concentrations at $n=n^{*}$ is fairly well verified. Of course these distributions are not as stable as true equilibrium distribution obtained in undersaturated alloys (cf. Figure 1) but the time scale of their evolution is so large, compared with the time necessary to stabilize them, that the expression "quasi steady-state" is fully justified, but they do evolve with time. On the contrary, for high supersaturations, although it might be possible to find a particular time such that the concentration of monomers is the same than in the equilibrium distribution predicted by Equation (2) and the concentration at $n^{*}$ is about half of the predicted one, in average the distribution is only approximately in agreement with the predicted one. And in addition such configurations are observed only for short period of times as they evolve very quickly. This difference of behaviour with low saturated alloys is of course enhanced by smaller values of $n^{*}$ which are not in favour of the capillary approximation. These more complex situations will be examined in section 4 .

\section{Figures 1 and 2}

\subsection{Frenkel's model of cluster gas}

If the range of atomic interactions can be truncated such that two clusters do not interact, then the total free energy per site of a cluster distribution can be rigorously described as the sum of free energies of all clusters plus the contribution of the configuration entropy of the cluster distribution or gas (also called entropy of mixing): 


$$
F_{\text {total }}=\sum_{n} C_{n} F_{n}-k_{B} T \ln (\Omega)
$$

$C_{n}$ is the concentration of clusters of size $n, \Omega$ is the partition function of the cluster gas. Within the approximation of Frenkel's model of cluster gas it is considered that, whatever its size, a cluster occupies only one site of the lattice, which is acceptable as long as $N_{0}<<N_{s}$ where $N_{s}$ is the total number of sites in the system containing $N_{0}$ solute atoms. Within this approximation $\Omega$ is defined by:

$$
\Omega=\frac{N_{s} !}{\prod_{k=1}^{\infty} n_{k} !\left(N_{s}-\sum_{k=1}^{\infty} n_{k}\right) !}
$$

where $n_{k}$ is the number of clusters containing $k$ solute atoms. The conservation of solute atoms imposes:

$$
\sum_{k=1}^{\infty} k n_{k}=N_{0} \text { or } \sum_{k=1}^{\infty} k C_{k}=C_{0}
$$

with $C_{k}=n_{k} / N_{s}$ and $C_{0}=N_{0} / N_{s}$. Using Stirling's approximation to simplify factorial terms, the total free energy per lattice site writes:

$$
\left(\frac{F_{\text {total }}}{N_{s}}\right)=\sum_{k}^{\infty} C_{k} F_{k}+k_{B} T\left\{\left(1-\sum_{k=1}^{\infty} C_{k}\right) \ln \left(1-\sum_{k=1}^{\infty} C_{k}\right)+\sum_{k=1}^{\infty} C_{k} \ln \left(C_{k}\right)\right\}
$$

The technique to minimize this expression versus cluster concentrations $C_{k}$ while accounting for Equation (5) can be found in different flavours in the literature but the base remains the Lagrange multiplicator method:

$$
\frac{1}{N_{s}} \frac{\partial F_{\text {total }}}{\partial C_{n}}+\lambda \frac{\partial C_{0}}{\partial C_{n}}=0
$$

The parameter $\lambda$ is set for a given value of $n$, the most convenient choice being $n=1$, then reintroduced in Equation (7) to obtain the general result:

$$
\left[\frac{\tilde{C}_{n}}{1-\sum_{k} \tilde{C}_{k}}\right]=\left[\frac{\tilde{C}_{1}}{1-\sum_{k} \tilde{C}_{k}}\right]^{n} \exp \left(-\frac{\Delta F_{n}}{k_{B} T}\right)
$$

Usually, the denominator $\left(1-\sum_{k} \tilde{C}_{k}\right)$ is neglected, which is consistent with the assumptions used to derive this expression, and in practice one uses the simplified Equation (2) instead of the complete result.

It is worth noting that this cluster gas model is not an ordinary model of alloy, capable to provide a ready-to-use approximation of the partition function or of the free energy of the 
system, contrary to other popular thermodynamical models. It only proposes a description of the total free energy of a binary alloy as the sum of two well-defined contributions, i.e. one coming from the individual structure of clusters (their free energy $F_{n}$ ) and the other one coming from their collective arrangement. This decomposition is correct (provided that two solute atoms belonging to two different clusters do not interact) but even if the values of $F_{n}$, can be calculated with a high accuracy, the collective effect is oversimplified in the original Frenkel's work.

\subsection{Discussion}

Whether the solution is under- or super- saturated, the notion of equilibrium concentration remains of great interest for both the CNT and the classical CD even if in practice these two methods use different ingredients for calculating the equilibrium concentrations of clusters. Indeed, various thermodynamical approximations can be used to calculate the driving force in Equation (1), while Equation (2) relies exclusively on the notion of cluster gas described above.

It is reminded that in classical CD the expression of the evaporation coefficient can be obtained from that of the simpler condensation coefficient through the rule of detailed balance, where the equilibrium concentrations are described according to Equation (2) (see [17] for instance, or [20] for a complete review of these questions). Therefore, the knowledge of this expression is absolutely fundamental for classical CD. Due to the necessary assumption of low solute concentration to establish Equation (2) it is commonly accepted that $\mathrm{CD}$ is strictly valid only in the limit of high dilutions although it can provide an acceptable approximation for common dilutions. This problem is likely to be even more acute for ordered precipitates than for pure precipitates due to their proportionally larger volume. This could explain the deviations observed between $\mathrm{CD}$ and $\mathrm{KMC}$ simulations of precipitation in $\mathrm{AlZr}$ alloys, which increase with the temperature and the solute concentration [18]. Therefore, it is necessary to reconsider Equation (2) to improve the domain of validity of CD.

Alternatively, if the equilibrium concentrations could be correctly obtained, for instance following the latter method, then the nucleation driving force and other useful quantities could be determined accurately then introduced into models using such quantities. However, to make possible this reverse process it is necessary to enhance the notion of cluster gas, in particular to remove the fundamental approximation it is built on (any cluster occupy only one lattice site, whatever its size). This is the goal of the next section.

\section{Accounting for the matrix frustration}

\subsection{Constant exclusion volume}

Before trying to account for the real volume of each cluster, an intermediate case worthy to consider is the simple case such that all clusters have the same volume $V$ but with $V>1$ ( $V$ is here an integer value measuring the number of lattice sites really occupied by the cluster). The partition function of the cluster gas can be decomposed into a product of independent functions $\Omega^{k}$, each member $k$ being related to a class of cluster. Consider the first class to arrange, i.e. $k=1$; at this stage it is not yet necessary to assume any relation between the rank $k$ and the number of solute atoms in a cluster of class $k$ since $V$ is a constant. 
After that $(i-1)$ classes have been arranged in the lattice, $\Omega^{i}$ writes:

$$
\Omega^{i}=V^{n_{i}}\left(\frac{N_{s}}{V}-\sum_{k=1}^{i-1} n_{k}\right) ! / n_{i} !\left(\left(\frac{N_{s}}{V}-\sum_{k=1}^{i-1} n_{k}\right)-n_{i}\right) !
$$

$N s$ is normalized by $V$ to introduce the factorial notation which will be further simplified using for instance Stirling's approximation. Finally the equivalent of equation (4) writes:

$$
\Omega=\prod_{i=1}^{\infty}\left(\frac{V^{n_{i}}}{n_{i} !}\right) \frac{\left(\frac{N_{s}}{V}-\sum_{k=1}^{i-1} n_{k}\right) !}{\left(\left(\frac{N_{s}}{V}-\sum_{k=1}^{i-1} n_{k}\right)-n_{i}\right) !}=\left(\frac{V^{\sum_{k=1}^{\infty} n_{k}}}{\prod_{i=1}^{\infty} n_{i} !}\right) \frac{\left(\frac{N_{s}}{V}\right) !}{\left(\frac{N_{s}}{V}-\sum_{k=1}^{\infty} n_{k}\right) !}
$$

Using the same method than previously leads to a result very similar to equation (8):

$$
\left[\frac{\tilde{C}_{n}}{1-V \sum_{k} \tilde{C}_{k}}\right]=\left\{\left[\frac{\tilde{C}_{1}}{1-V \sum_{k} \tilde{C}_{k}}\right]^{\mathrm{n}} \exp \left(-\frac{\Delta F_{n}}{k_{B} T}\right)\right\}
$$

Continuing to apply the same approach for the general case was found too confusing to lead to a practical solution without extensive simplifications. Instead, it is much easier to use the fact that when the cluster gas is at equilibrium, by definition, an elementary change in the concentration of any class does not change the total free energy. The simplest perturbation to consider consists in removing a cluster of a given class and then to redistribute its solute atoms among other classes. Any redistribution is acceptable but one of them is of particular interest: the dissolution of the cluster of size $n$ into $n$ individual monomers. This choice is the only one which reduces the problem to the examination of the linked evolution of two classes, 1 and $n$ (now the rank is also the number of solute atoms in the cluster). In practice, the problem amounts to compare the partition function before and after the transformation of the $n$-mer into $n$ monomers, or even easier, to compare the partition function after introduction of the last $n$ solute atoms, following the two different modes.

The intermediate case treated above is used to illustrate this method. After removing the terms in common, the ratio of the new and old partition functions (i.e. after and before the dissolution of the $n$-mer) writes:

$$
\left(\frac{\Omega_{\text {new }}}{\Omega_{\text {old }}}\right)=V^{k-1}\left(\frac{n_{1} !}{\left(n_{1}+k\right) !}\right)\left(\frac{n_{k} !}{\left(n_{k}-1\right) !}\right) \frac{\left(\frac{N_{s}}{V}-\sum_{i=1}^{\infty} n_{i}\right) !}{\left(\frac{N_{s}}{V}-\sum_{i=1}^{\infty} n_{i}-(k-1)\right) !}
$$


The total variation of free energy per site writes:

$$
\Delta F_{\text {total }}=-k_{B} T\left\{\left(\frac{\Delta F_{n}}{k_{B} T}\right)+\ln \left(\frac{\Omega_{\text {new }}}{\Omega_{\text {old }}}\right)\right\}=0
$$

The logarithmic term can be estimated following the same calculation scheme than previously, which gives:

$$
\left(\frac{\Omega_{\text {new }}}{\Omega_{\text {old }}}\right)=\left(\frac{\tilde{C}_{k}}{1-V \sum_{i} \tilde{C}_{i}}\right)\left(\frac{\tilde{C}_{1}}{1-V \sum_{i} \tilde{C}_{i}}\right)^{-k}
$$

It can be easily checked that reporting this expression into Equation (15) leads again to Equation (12).

It is worth noting that the key hypothesis required to establish this result is not related to the solute dilution but is the simple condition $n_{l}>>k$, where $k$ is any cluster size of the considered distribution. In other words, it assumes that the number of monomers is very large compared to the number of solute atoms in the largest cluster to be considered, a condition always fulfilled in situations of practical interest. The alloy dilution can play a role only in the required degree of approximation of the factorial terms. However, as Equation (13) is a ratio of pairs of very similar factorial terms, it can be easily checked that the result is not sensible to the approximation used for factorials; in practice Stirling's approximation is sufficient. Finally, the only restriction is trivial: the description of the solute phase in terms of clusters or isolated precipitates has to be relevant with regards to the solute concentration, i.e. this description assumes that clusters do not percolate.

\subsection{Real exclusion volume}

To apply this simple method to the complex general case it is convenient to define the quantities $M_{k}$, the respective numbers of sites available to insert a new $k$-mer in the system. With $V_{j, i}$ the number of sites that a cluster of size $j$ forbids to a cluster of size $i$, we have:

$$
M_{i}=N_{s}-\sum_{j} V_{j, i} n_{j}
$$

Then we need to generalize Equation (11). Following the same reasoning way than for mono-size clusters, we list all the possible states of the system, accounting now for the real values of exclusion volumes $V_{j, i}$

$$
\Omega=\prod_{i=1}^{\infty}\left(\frac{V_{i, i}^{n_{i}}}{n_{i} !}\right) \frac{\left(\frac{M_{i}^{*}}{V_{i, i}}\right) !}{\left(\frac{M_{i}^{*}}{V_{i, i}}-n_{i}\right) !}
$$


Here $M_{i}^{*}$ refers to the number of sites left available by classes of clusters already inserted in the system, see Equations (9-11). Contrary to Equation (11) this result cannot be simplified anymore because of the different values $V_{j, i}$. It is reminded that the use of the factorial notation is intended to compact the result and facilitates its calculation, but it is important to not forget the original form analogous to Equation (9). Indeed, developing the full result it is easy to see that the value of $\Omega$ given by Equation (17) depends now on the order of introduction of the various clusters. As it is a purely mathematical procedure and not a physical process, only the maximal value of $\Omega$ should be retained. This maximal value corresponds roughly to the introduction of the different classes of clusters in the inverse order of their size, i.e. first the largest clusters and finally the monomers. Equation (17) can be rewritten in a polynomial form:

$$
\Omega=N_{s} \sum_{k=1}^{N_{c}} a_{k} N_{s}^{N_{c}-k} / \prod_{i=1}^{\infty} n_{i} !
$$

Here $N_{c}$ is the total number of clusters $\left(N_{c} \leq N_{0}\right)$ and the coefficients $a_{k}$ are complex functions of the exclusion volumes and concentrations but the two first values are special cases. The first coefficient, $a_{1}=1$ in any cases and $a_{2}$,which is easy to calculate only if all exclusion volumes are identical, is a constant for a given system, i.e. it does not depend on the way to build the cluster gas contrary to the following coefficients. However, in principles, it is always possible to consider a system large enough to ensure that $\left|a_{k} N_{s}\right|>>\left|a_{k+1}\right|$ for $k>2$. By consequent, the value of $\Omega$ given by Equation (17) can be considered independent on the (virtual) order of introduction of the various clusters, as in the simplest case, at least for large systems. If, as in the present paper, one plans to compare with simulation results obtained with finite size systems it is worth to refine these considerations about the expression of $\Omega$. Hopefully, with the method introduced in the previous section we do not really need to evaluate $\Omega$, we only need to evaluate variations of $\Omega$. Coming back to the introduction of the last $n$ solute atoms in the system either under the form of $n$ monomers or one $n$-mer, we can take as common reference the initial value of $\Omega$, assumed exact Then even for a finite size system it can be reasonably assumed that the two modes of introduction of the last $n$ solute atoms do not require $\Omega$ to be evaluated again (it is reminded that $n$ is implicitly assumed much smaller than the total number of solute atoms in the system). Therefore, the equivalent of Equation (13) writes:

$$
\left(\frac{\Omega_{\text {new }}}{\Omega_{\text {old }}}\right)=\left(\frac{V_{1,1}^{k}}{V_{k, k}}\right)\left(\frac{n_{1} !}{\left(n_{1}+k\right) !}\right)\left(\frac{\left(n_{k}+1\right) !}{n_{k} !}\right) \frac{\left(\frac{M_{1}}{V_{1,1}}\right) !}{\left(\frac{M_{1}}{V_{1,1}}-k\right) !} \frac{\left(\frac{M_{k}}{V_{k, k}}-1\right) !}{\left(\frac{M_{k}}{V_{k, k}}\right) !}
$$

Assuming the trivial conditions $M_{1}>>k V_{1,1}$ and $M_{k}>>V_{k, k}$, and applying the same method than for the intermediate case (constant $V$ ), the equivalent of Equation (15) writes:

$$
\left(\frac{\Omega_{\text {new }}}{\Omega_{\text {old }}}\right)=\left(\frac{\tilde{C}_{k}}{M_{k}}\right)\left(\frac{\tilde{C}_{1}}{M_{1}}\right)^{-k}=\left(\frac{\tilde{C}_{k}}{1-\sum_{i} V_{i, k} \tilde{C}_{i}}\right)\left(\frac{\tilde{C}_{1}}{1-\sum_{i} V_{i, 1} \tilde{C}_{i}}\right)^{-k}
$$


Finally, the general solution takes a form which could be guessed from the structure of equation (12):

$$
\left[\frac{\tilde{C}_{n}}{1-\sum_{k} \tilde{C}_{k} V_{k, n}}\right]=\left[\frac{\tilde{C}_{1}}{1-\sum_{k} \tilde{C}_{k} V_{k, 1}}\right]^{n} \exp \left(-\frac{\Delta F_{n}}{k_{B} T}\right)
$$

If all exclusion volumes are equal, both intermediate and classical solutions are readily obtained (i.e. Equations (12) and (8), respectively).

It can be objected that this results is valid only for small values of $n$ because the dissolution of a large cluster cannot be considered an elementary change of the partition function. Indeed, the most elementary change would be, as in cluster dynamics, the transformation of an $n$-mer into an (n-1)-mer plus a monomer. Using the same technique than above, the ratio of the partition functions corresponding to these two states of the system writes now:

$$
\left(\frac{\Omega_{\text {new }}}{\Omega_{\text {old }}}\right)=\left(\frac{V_{1,1} V_{k-1, k-1}}{V_{k, k}}\right)\left(\frac{\left(n_{k}+1\right)}{\left(n_{k-1}+1\right)\left(n_{1}+1\right)}\right) \frac{\left(\frac{M_{1}}{V_{1,1}}\right) !}{\left(\frac{M_{1}}{V_{1,1}}-1\right) !\left(\frac{M_{k-1}}{V_{k-1, k-1}}-1\right) !} \frac{\left(\frac{M_{k-1}}{V_{k-1, k-1}}\right) !}{\left(\frac{M_{k}}{V_{k, k}}\right) !}
$$

After simplification, with $\bar{M}_{n}=M_{n} / N_{s}$, the equivalent of Equation (20) for this transition writes:

$$
\left(\frac{\Omega_{\text {new }}}{\Omega_{\text {old }}}\right)=\left(\frac{\tilde{C}_{k}}{\bar{M}_{k}}\right) /\left(\frac{\tilde{C}_{1}}{\bar{M}_{1}}\right)\left(\frac{\tilde{C}_{k-1}}{\bar{M}_{k-1}}\right)
$$

The drawback of this equation is that it couples the three classes $n,(n-1)$ and 1 . But there is a particular case for which Equations (20) and (23) are strictly equivalent: the transformation of 2-mer into two monomers. Thus, setting $k=2$ in Equation (23) leads to the same result than Equation (21) for 2-mers. Then, by recurrence, incrementing $k$ in Equation (23) leads again to Equation (21) for all possible classes of cluster sizes, which validates $a$ posteriori the initial choice about the simplest (if not the most elementary) configuration change to consider. Note that after posing $\widehat{C}_{n}=\left(\widetilde{C}_{n} / \bar{M}_{n}\right)$ equation (21) can be rewritten in a compact notation analogous to Equation (2).

Although Equation (21) is now fully justified, there are still hidden hypothesis which deserve to be addressed. To compute the equilibrium concentrations, section 3.3 address the definition of the exclusion volumes $V_{j, i}$ entering in the definition of terms $M_{n}$. Finally the section 3.4 will show that Equation (21) is still an approximation for very dilute alloys: indeed, neglecting the multi-exclusion in Equation (16), i.e. the fact that the various exclusion volumes can superimpose is likely to overestimate the total exclusion volume at high solute concentration. After some further developments it will be shown how Equation (21) can be rewritten in a general form. 


\subsection{Individual exclusion volume}

It is recalled that, by definition, a cluster is surrounded by a layer free of solute, then by a second layer from where solute atoms can be captured of released, the exact shape of these two layers changing after each events (cf. paper I). In addition to their industrial interest, AlZr alloys are good candidates to test the present model due to their low solubility, which allows for large supersaturations, and the $\mathrm{L}_{12}$ structure of the $\mathrm{Al}_{3} \mathrm{Zr}$ precipitates, which is likely to enhance the frustration effect. In addition, with atomic interactions limited to $\langle 100\rangle$ (second) neighbours, due to the strong repulsive force between $\mathrm{Zr}$ atoms in position of first neighbours, the first neighbouring $<110>$ is constant for all $\mathrm{Al}$ and $\mathrm{Zr}$ atoms [3]. Thus, some results given in paper I for the cubic structure are directly applicable, in particular the free energy surface term. Others, like the numbers of sites in the first shell are peculiar to the $\mathrm{L}_{12}$ structure and in this case, compared with pure cubic clusters, the f.cc. structure of the matrix tends to smooth such properties of $\mathrm{L}_{12}$ clusters.

For large clusters the particular value $V_{n, 1}$ is easy to estimate: $V_{n, 1} \approx n / C p$ where $C p$ is the atomic solute concentration of clusters $\left(C p=1 / 4\right.$ for $\mathrm{L}_{12}$ clusters). Indeed, for large clusters the relative importance of surface sites versus volume sites becomes more and more negligible as their size increases. The total volume of a cluster (i.e. including the solute free layer) is nothing but the exclusion volume for monomers. This quantity was calculated following the Monte Carlo method described in paper I as a function of the reduced temperature $T_{w} / T$ where $T_{w}$ characterizes the material at temperature $T$. The two first values of $\left(V_{n, l} / n\right)$ being independent on $T_{n} / T$, only the rate of convergence toward the asymptotic limit can depend on this parameter. This result is plotted on Figure 3; it shows that $\left(V_{n, 1} / n\right)$ decreases very quickly at the beginning then converges toward its asymptote as expected, more rapidly at low temperature than at high temperature.

Then for $k>1, V_{n, k}$ is estimated by approximating the excluded volume by a sphere whose radius is the sum of the radii of the two clusters, also approximated as spheres as shown in Figure 4.

$$
V_{i, j}=\frac{4 \pi}{3}\left(R_{i}+R_{j}\right)^{3}
$$

First, the comparison with $V_{l, l}$ provides $R_{l}$, then the comparison with $V_{k, l}$ (for $k>1$ ) gives the following values of $R_{k}$. These effective radii are represented in Figure 5. The exact calculation with the Monte Carlo method used for calculating $V_{n, 1}$ would require a complex sampling and thermal averaging procedure over the shapes of both clusters I and J. Then the result should be translated into a form easy to manipulate like Equation (24). In principles, the task is not impossible, but it is very doubtful that this complex calculation would significantly modify the values of $V_{i, j}$ derived from Equation (24) and the accurate calculation of $V_{n, 1}$.

\section{Figures 3 and 4}

As it can be seen in Figure 5, the value of clusters effective normalized radii do not depend or are only weakly dependent on the ratio $T_{w} / T$ for the few first classes, contrary to larger clusters. Like other quantities at low temperature, $R_{n}$ exhibits some peaks, valleys and stairs: it is a consequence of the existence of compact configuration for particular values of $n$ (the best example is $n=8$ ). For intermediate temperature (i.e. $723 \mathrm{~K}$ for AlZr alloys) these fluctuations almost vanish and at higher temperature the curves are smooth. The lower the temperature the closer remains $R_{n}$ from its asymptotic limit. The increasing values of $R_{n}$ at high temperature are mostly due to the roughness of the cluster surfaces by contrast to the sharp surfaces 
observed at low temperature. The shape factor investigated in paper I does not play any role here. For numerical applications (section 4.2) these quantities have been fitted to get the values of $R_{n}$ when $\mathrm{n}>100$. The fitting procedure has been described in details in paper I. Briefly, it is established from calculated values in the range $35<n<100$, typically, then compared with few values calculated in the range $100<n<400$. With this procedure, accurate and stable fits (relative accuracy of the order of $10^{-3}$ ) were obtained for the range $n>35$ with only few parameters (cf. Table 1). No accurate and stable fit was found for the range $2<n<35$, at least not with simple expressions, thus computed values were used for numerical applications presented in the next section.

\section{Table 1}

Figure 5

\subsection{Accounting for multiple exclusion}

The knowledge of these individual exclusion volumes is sufficient for very dilute alloys but even for concentrations of the order of $1 \%$, to avoid the overestimation of the exclusion effect (i.e. underestimation of the partition function $\Omega$ ) it is necessary to express the terms $M_{n}$ in a very general way, able to account for multiple exclusions. The meaning of $M_{n}$ and the expression of the equilibrium concentrations are preserved; only the numerical values of terms $M_{n}$ are modified by multi-exclusion.

Consider the status of a particular site versus the exclusion of clusters of size $j$ by clusters of size $i$. This site can be excluded by 1,2 or more clusters $i, i$ varying over all possible classes of cluster size. Expressing these different probabilities is rather complex due to coupling effects, especially for an ordered structure. But this knowledge is not necessary; indeed, one only needs to know the probability for a given site to not be excluded, which is much simpler. Basic combinatorial probability analysis leads to:

$\bar{M}_{n}=\prod_{k}\left(1-C_{k}\right)^{V_{k, n}}$

Substituting this result to Equation (16) into Equation (21) gives the generalized solution for equilibrium concentrations:

$\tilde{C}_{n}=\tilde{C}_{1}^{n} \exp \left(-\frac{\Delta F_{n}}{k_{B} T}\right)\left(\frac{\bar{M}_{n}}{\bar{M}_{1}^{n}}\right)=\tilde{C}_{1}^{n} \exp \left(-\frac{\Delta F_{n}}{k_{B} T}\right) \prod_{k}\left(1-\tilde{C}_{k}\right)^{\left[V_{k, n}-n V_{k, 1}\right]}$

For dilute alloys such that $C_{k}<<1$ for all values of $k$, Equation (25) simplifies:

$\bar{M}_{n}=\exp \left(\sum_{k} V_{k, n} \ln \left(1-C_{k}\right)\right) \approx \exp \left(-\sum_{k} V_{k, n} C_{k}\right)$

Consequently Equation (26) takes a much simpler form, with $\Delta V_{k, n}=\left(V_{k, n}-n V_{k, 1}\right)$ :

$\tilde{C}_{n}=\tilde{C}_{1}^{n} \exp \left(-\frac{\Delta F_{n}}{k_{B} T}\right) \exp \left(-\sum_{k} \tilde{C}_{k} \Delta V_{k, n}\right)$ 
Equation (28) is equivalent to Equation (21) only for high dilutions; indeed, in this case, Equation (27) simplifies, i.e. one finds again Equation (16).

When $C_{k}$ is not small enough to apply the above simplification, typically for $k=1$ or less probably $k=2$ or even 3 , the respective terms $\left(1-\tilde{C}_{k}\right)^{\Delta V_{k, n}}$ has to be introduced following the form of Equation (26), then the form of Equation (28) can be applied for the following terms. In other words, the cumbersome form of Equation (26) can always be avoided, except for the very few first terms if the solute concentration is high. This also shows that depending on the alloy concentration and the approximation level adopted to describe the cluster gas, the equilibrium concentrations can be easily under- or over- estimated.

Note that whatever the level of approximation (i.e. Equation (21) or (26) or (28)), the key quantities controlling this frustration effect are the factors $\Delta V_{k, n}$ plotted in Figure 6 for a few values of $n$. The quantity $\Delta V_{k, n}$ increases with both $n$ and $k$. And for large values of $n$ and $k$, since $V_{k, n}=V_{n, k}$ and $\left(V_{1, n} / n\right)$ has an asymptotic behaviour we have $\Delta V_{k, n} \approx \Delta V_{n, k}$ which explains why all curves seem to be parallel. Note that even in the represented $k$ range in Figure 6 the different curves are very similar and most of all they were found to be almost independent on the parameter $T_{w} / T$, which is coherent with the way $V_{k, n}$ is built for $k>1$. This can be understood as a measure of the sensibility of clusters of size $\mathbf{J}$ to explore the structure of clusters of size I: obviously, whatever the value of I, monomers are the most efficient probes and the sensibility decreases with $\mathrm{J}$.

\section{Figure 6}

\section{Applications}

\subsection{Introduction of the matrix frustration effect in $C D$}

As recalled in introduction, whatever the method chosen to solve the master equation of CD, i.e. with one (I) or two (II) pairs of coefficients, the key quantity is the ratios of the partition function of the system before and after the capture or the release of a solute atom by a cluster of size $n$. When the matrix frustration is neglected this amounts to consider the quantity $\exp \left(-\left(\Delta F_{n+1}-\Delta F_{n}\right) / k_{B} T\right)$. To remain general, the coefficients of absorption (I), or capture (II), and emission (I), or release (II), will be noted $w_{n, n+1}$ and $w_{n+1, n}$, respectively. Accounting for the frustration effect can be readily done by application of the detailed balance:

$w_{n, n+1} \tilde{C}_{n}=w_{n+1, n} \tilde{C}_{n+1}$

Then, using equation (28) to express the equilibrium concentrations, it comes:

$$
\frac{w_{n, n+1}}{w_{n+1, n}}=\exp \left(-\frac{\Delta F_{n+1}-\Delta F_{n}}{k_{B} T}\right) \exp \left(-\sum_{k} \tilde{C}_{k}\left(\Delta V_{k, n+1}-\Delta V_{k, n}\right)\right)
$$

The only difference between schemes I and II is that this ratio appears only in the emission coefficient in the classical method (the usual expression is limited to the first term) while its contribution to capture and release coefficients in method II is symmetrical. Notations introduced in paper I and entering in the definition of the capture and release coefficients are first recalled: 


$$
\begin{aligned}
& \bar{P}_{n, n+1}^{0}=v\left[\frac{T_{n, n+1}}{\sqrt{Q_{n}^{*} Q_{n+1}^{*}}}\right] \sqrt{\frac{Q_{n+1}^{*}}{Q_{n}^{*}}} \\
& \bar{P}_{n, n+1}^{0}=v\left[\frac{T_{n, n+1}}{\sqrt{Q_{n}^{*} Q_{n+1}^{*}}}\right] / \sqrt{\frac{Q_{n+1}^{*}}{Q_{n}^{*}}}
\end{aligned}
$$

Original equations have been rewritten to better put into evidence the symmetry of these two coefficients. $T_{n, n+1}$ is the transition function between states $n$ and $n+1, Q_{n}^{*}$ is a reduced partition function of clusters such that $Q_{n}^{*}=\exp \left(-\Delta F_{n} / k_{B} T\right)$; the attempt frequency $v$ does not play any role in the present problem. Finally, with $C_{n}^{\text {shell }}$ the concentration of solute atoms in the second shell of clusters of size $n$, taking the contribution of cluster gas entropy symmetric like the contribution of cluster internal entropy in Equations (27-28) (the partition functions $Q^{*}$ and $\Omega$ are independent), the coefficients of emission or absorption for scheme II can be fully defined:

$$
\begin{aligned}
& P_{n, n+1}=\bar{P}_{n, n+1}^{0} \sqrt{\exp \left(-\sum_{k} C_{k}\left(\Delta V_{k, n+1}-\Delta V_{k, n}\right)\right)} C_{n}^{\text {shell }} \\
& P_{n+1, n}=\bar{P}_{n, n+1}^{0} / \sqrt{\exp \left(-\sum_{k} C_{k}\left(\Delta V_{k, n+1}-\Delta V_{k, n}\right)\right)}
\end{aligned}
$$

Note that all concentrations in the corrective term are now real concentrations, but of course, at equilibrium the ratio of these two coefficients verifies the equality which is the base of classical CD: $\left(P_{n, n+1} / P_{n+1, n}\right)=\left(\tilde{C}_{n+1} / \tilde{C}_{n} \tilde{C}_{1}\right)$.

\subsection{Comparison of predicted distributions with those obtained in KMC simulations}

The best test to investigate the validity of the proposed solution is to compare the predicted distributions of clusters with those obtained in KMC simulations, a technique completely independent of the present formalism, at the opposite of a cluster dynamics algorithm which would be based on Equation (33-34). Results obtained by Clouet [16-17] for AlZr at 723K $\left(C_{0}=1 \%\right)$ and $873 \mathrm{~K}\left(C_{0}=1 \%\right.$ and $\left.3 \%\right)$ were selected for this test.

The first point to clarify is the nature of such a comparison, i.e. what should be compared and in what conditions, knowing that KMC simulations can provide cluster distributions at various times along the precipitation kinetics. Apparently the problem is trivial, such comparisons becoming more common in the literature. But the lack of details about the comparison procedure reveals that the difficulty of such comparisons has been underestimated.

In absence of frustration, a particular case consists in calculating the equilibrium concentrations such that all solute atoms are contained in the subcritical classes, i.e. $n \leq n^{*}$. It is the solution usually adopted to define the initial value of $n^{*}$, and used for instance to differentiate "precipitates" from the "solid solution" in KMC simulations referred to for this test. This criterion defines a particular value of $C_{l}$, the concentration in monomers, which is reached at a particular time in KMC simulations further used to define the first set of curves in 
Figures (7-9). For different times, one has to adopt a reverse procedure: first choosing a given distribution from KMC simulations, which in return defines a particular value of $C_{l}$, then one has to impose this value as the new and only constraint to calculate the cluster distribution, truncated at $n^{*}$ as previously. In this case, the total concentration in solute is disregarded; indeed, only the concentrations in subcritical classes can be directly compared with real or simulated ones, by definition (it is reminded that in the case of true equilibrium as in Figure 1, all classes are subcritical).

To account for the frustration effect one has to adopt a similar procedure, but contrary to the previous case, the concentration of a given class depends also on the concentrations of other classes, not only on $C_{l}$. It is of course possible to self-consistently evaluate such a distribution (an application is proposed in section 4.4), but like the solution without frustration, it diverges beyond the critical size, contrary to real or KMC distributions. And if the distributions are truncated at $n^{*}$ the comparison becomes biased by construction. Thus, the most physically significant comparison consists in calculating the frustration effect in Equation (28) according to the KMC distribution one wants to compare with. It is precisely this comparison procedure which revealed the limits of the approximated solution (Equation 21 ), the exclusion volume becoming larger than the system volume beyond a certain cluster size.

Figures (7-9) compare these two solutions, with and without frustration, with the KMC distributions of same $C_{l}$ for three different times. The first one, $t_{l}$, as defined above, while the second time, $t_{2}$, corresponds to the smallest time such that the solution with frustration coincides fairly well with the KMC distribution up to $n^{*}$. This agreement is preserved approximately until the number of precipitates $N_{p}(t)$ starts to decrease. Figure (10) proposes a schematic representation of the different stages observed along the precipitation kinetics of these aluminium alloys. Note that the different stages of the curve $C_{l}(t) / C_{0}$ do not strictly coincide with the well-known stages of the evolution of the average size of clusters, not reported here (see [17]). For the scope of the present paper we are mostly concerned with the long intermediate stage which corresponds roughly with the so-called nucleation stage. Beyond the maximum of $N_{p}(t)$ the agreement between $\mathrm{KMC}$ and predicted distributions gradually deteriorates, at least for large (subcritical) classes while it is well preserved for the small classes. The third set of curves in Figure 7 and 9 corresponds to a time $t_{3}$ taken near the transition from the third to the fourth stage of $C_{l}(t) / C_{0}$ in Figure (10), i.e. well after the maximum of $N_{p}(t)$. For technical reasons, in Figure 8, this third set corresponds to a time taken just before this transition (due to the low supersaturation in this case, the number of clusters in supercritical classes becomes too small for a reliable comparison). We now discuss this comparison for these three set of curves.

Time $t_{1}$ always corresponds to early nucleation, as expected, but note that it might correspond to very different values of $C_{l}(t) / C_{0}$ depending on $C_{0}$ and the ratio $T_{w} / T$. Various situations are observed:

- Figure 7: similar to the ideal situation shown in figure 2, the ratio 2 between the predicted concentration at $n^{*}=7$ and the simulated one is fairly well verified; the distributions are in reasonable agreement up to $n=5$. Thus at first sight this situation appears very classical. The solution with frustration exhibits the same $n^{*}$ than the simplest solution, is in excellent agreement up to $n=3$ but overestimates the concentrations in the following classes.

- Figure 8: $n^{*}=25$ while the KMC distribution extends only up to $n=14$ (a huge simulation box would be necessary to occupy all classes up to $n=25$ for such a short time). Consequently the solution without frustration seems to be the best one. 
Compared with the previous case the two solutions are now well separated, both in $n^{*}$ and $C_{n}$ values.

- Figure 9: the KMC distribution is much larger than the predicted critical sizes $\left(n^{*}=5\right.$ and 8 , with and without frustration, respectively) but for low values of $n$ it is clearly in between the two predicted solutions. Again, compared with the first case, note that the two solutions are well dissociated. The ratio of concentrations at $n^{*}$ (without frustration) is here about 3.

This first set of curves does not provide any clear advantage for one solution over the other, but shows that the situation is effectively much more complex than the ideal picture shown in Figure 2 and is very dependent on both the solute concentration and the parameter $T_{n} / T$ (or more simply the temperature for a given material). To understand these differences of behaviour it is necessary to examine how these distributions evolve during the precipitation kinetics.

At $t=0$, the distributions are mostly composed of monomers; other small clusters are in so small concentration than the associated curve would be almost a vertical line in Figures (7-9). When a cluster distribution builds on, its length and concentrations in all classes, except the first one, increase; later, the concentrations of small clusters start to decrease too. Thus, at the very beginning of precipitation, it is not surprising to find the KMC curves under the predicted ones except for the very first subcritical classes.

Consider now the ability of the two predicted solutions to follow the evolution of the KMC distributions when time increases. Excellent agreement between KMC distributions and the predicted solution without frustration is never observed and when this agreement is, say acceptable, it is observed only during a very short period of time. Much better agreement is observed with the solution accounting for frustration, but this agreement needs more time to be reached. However, contrary to the solution without frustration which increasingly deviates, this agreement with KMC distributions improves with time and finally concerns the whole curve (at time $t_{2}$ ). In addition, this agreement is well preserved until time $t_{3}$ when the cluster distributions become increasingly influenced by the growth and coarsening processes versus nucleation, except for small classes. Indeed, while undersaturated alloys exhibit Boltzmannlike cluster distributions (Figure 1), in supersaturated alloys, with time, cluster distributions are known to evolve towards a Lifshitz - Slyozov - Wagner-like distribution [20]; hence intuitively one expects large subcritical classes to deviate from the Boltzmann-like distribution after some annealing time. The analysis of the two most supersaturated cases studied here (Figures 7 and 9) suggests a naïve criterion: the divergence between predicted and simulated distributions appears when the fraction of solute in supercritical classes approaches (and then exceeds) that contained in subcritical classes. Of course, this criterion does not refer to a sharp transition; in one hand the agreement is not absolutely perfect up to that point and in the other hand it remains acceptable for a while beyond this transition, which corresponds fairly well with the end of the nucleation stage. Another argument is that the agreement between predicted and KMC distributions is clearly better or at least easier to put into evidence for the case leading to the largest $n^{*}$, which corresponds to the least supersaturated case (Figure 8).

Note on figure 9 that two curves are almost superimposed, but correspond to different solutions and times, and by consequent different $C_{l}$ : this stresses the importance of defining carefully the conditions of such comparisons. Similar comparisons can be found in the literature for other materials, see [4] for instance. The results for high dilutions look similar to presents ones, however, as the criterion applied to perform the comparison is different (the 
equality of $C_{1}$ is not enforced), the main divergence between predicted and KMC distributions localizes on low classes while the rest of the curves seems to be in approximate agreement.

From the comparison of these three figures, no simple criterion can be derived to easily predict the influence of the frustration effect without any calculation. Indeed, at least two parameters are necessary to describe the observed behaviour: the ratio $T_{w} / T$ which controls the free energy of clusters and the total solute concentration which controls the magnitude of the exclusion effect. Alternatively, one could also adopt parameters derived from these fundamental quantities, e.g. the solubility limit and the supersaturation. The key quantity responsible of this complexity is certainly the free energy of clusters calculated in paper I, a quantity whose evolution with $T_{w} / T$ can be considered fairly self-similar only in a very restricted range. For AlZr alloys considered here, the free energy at $873 \mathrm{~K}$ is quite different from that at $723 \mathrm{~K}$, a temperature for which the free energy is typical of this intermediate range.

\section{Figures 7-9}

Comparing the magnitudes of cluster concentrations for the different KMC curves reported here with the magnitudes of exponents $\left(V_{k, n}-k V_{n, 1}\right)$ reported in Figure (6) shows that the contribution of the different classes of size $k$ to the frustration effect concerning a class $n$ decreases with $k$, as expected; but note that this decreasing is fairly slow for Figures 7 and 9 compared with Figure 8 which corresponds to a much lower supersaturation. In the latter case, the calculation of the frustration effect could be based only on subcritical classes without noticeable differences in predicted distributions. This contributes to explain why classical models based on the CNT framework (including classical CD) might be successful for low saturated alloys but increasingly deviate with increasing concentrations, generally speaking.

\section{Figure 10}

\subsection{Critical cluster size}

Consider a cluster of size $n$, it is in equilibrium with its interface if the probabilities to capture or to release a solute atom are equal, i.e. if $P_{n, n+1}=P_{n, n-1}$. According to Equations (34-35) this condition is achieved if the solute concentration in the shell equals a critical value $C_{n^{*}}^{\text {shell }}=\left(\phi_{n, n-1} T_{n, n-1} / \phi_{n, n+1} T_{n, n+1}\right)$. If $C_{n^{*}}^{\text {shell }}<\tilde{C}_{1}$ the local concentration of solute atoms in the shell will increase by diffusion from the matrix and the cluster will grow up until it reaches a new size in equilibrium with the average concentration of monomers. Inversely, if $C_{n^{*}}^{\text {shell }}>\widetilde{C}_{1}$, the cluster will shrink until it reaches its new equilibrium size. Thus, one can associate a critical value $n^{*}$ with the concentration of monomers, such that:

$$
\tilde{C}_{1}=\left(\frac{\bar{P}_{n^{*}, n^{*-1}}^{0}}{\bar{P}_{n^{*}, n^{*}+1}^{0}}\right) / \exp \left(\sum_{k} C_{k}\left(V_{k, 1}+\left(V_{k, n^{*}-1}-V_{k, n^{*}+1}\right) / 2\right)\right)
$$

Strictly speaking, solving this equation requires the knowledge of both the capture and release rates as reported in Figure 11 and 12, respectively. These results are comparable with those obtained for pure cubic clusters (cf. Paper I), however these coefficients depend also on the structure of the matrix, the magnitudes of coefficients are different and at low temperature the strong oscillations are smoother. As it can be seen in Figure (11), the release factor varies 
slowly enough to reasonably assume that $\bar{P}_{n, n-1}^{0} \approx \bar{P}_{n+1, n}^{0}$, which allows for some simplifications: $C_{n^{*}}^{\text {shell }} \approx \tilde{C}_{n^{*}} \tilde{C}_{1} / \tilde{C}_{n^{*}+1}$ then the equilibrium condition $C_{n^{*}}^{\text {shell }}=\tilde{C}_{1}$ becomes $\tilde{C}_{n^{*}}=\tilde{C}_{n^{*}+1}$. Classically, the observed minimum on the curve $\tilde{C}_{n}$ is assumed to define the critical size $n^{*}$. This conclusion arises from a similar reasoning way but in a continuous space of cluster size. Comparing this approximation with Equation (35) and KMC distributions did not reveal deviations larger than few units (i.e. the condition (35) can be reached before the minimum of the distribution). Thus in first approximation, this classical approximation is justified; nevertheless, its application to alloys of high concentration should be considered with great care. Indeed, even such a small difference is likely to induce important differences in the predicted kinetics of precipitation.

\section{Figures 11-12}

\subsection{Nucleation driving force in CNT}

It is interesting to analyse what can be learnt from this work when compared with classical models based on the CNT formalism (see for instance [20-21]). In the CNT framework the formation free energy $\Delta G_{n}$ of $\mathrm{L}_{12}$ clusters writes:

$\Delta G_{n}=4 n \Delta G_{n}^{n u c}+F_{n}$

Here $\Delta G_{n}^{n u c}$ is the nucleation free energy for clusters containing $n$ solute atoms; it can be deduced from the comparison of equations (1) and (28):

$4 n \Delta G_{n}^{n u c}=-n F_{1}-k_{B} T\left(n \ln \left(\tilde{C}_{1}\right)+\sum_{k} \tilde{C}_{k}\left(n V_{k, 1}-V_{k, n}\right)\right)$

$F_{l}$ depends on the temperature and the order energy $W_{a b}$, function of bond energies (cf. paper I), finally $\left(F_{1} / k_{B} T\right)=6\left(T_{w} / T\right)$ where the factor 6 holds for the coordination of the cubic sublattice. With these notations, $\Delta G_{n}^{n u c}$ writes:

$$
\Delta G_{n}^{n u c}=-\left(\frac{k_{B} T}{4}\right)\left\{6\left(\frac{T_{w}}{T}\right)+\left(\ln \left(\tilde{C}_{1}\right)+\frac{1}{n} \sum_{k} \tilde{C}_{k}\left(n V_{k, 1}-V_{k, n}\right)\right)\right\}
$$

In the CNT framework $\Delta G_{n^{*}}^{n u c}$ has to be determined for the value of $n$ which minimizes $\Delta G_{n}^{n u c}$. By construction this criterion is equivalent to choose the minimum of the curve $\tilde{C}_{n}$ which coincides with or is close to the critical value $n^{*}$, as shown in the previous section. In practice, when disregarding the frustration effect, the calculation of $\tilde{C}_{n}$ depends only on $\tilde{C}_{1}$, while when accounting for this effect it depends on concentrations of all classes. In the former case, two situations are possible whether real or KMC distributions are available or not. When such distributions are available, we saw in the previous section that the most physical calculation of equilibrium concentrations consists in accounting for the whole distribution. If no reliable distribution is available one can still calculate equilibrium 
concentrations following a self - consistent scheme. To estimate the relevance of this procedure, a comparison between both calculation modes is presented.

Consider first the simplest calculation mode, which is very similar to the case without frustration effect. To obtain accurate values, the length of the distribution was fixed and the simplified criterion to determine $n^{*}$ described above was applied, i.e. the equality of the two last equilibrium concentrations was imposed $\left(n^{*}\right.$ and $\left.n^{*}+1\right)$, giving the corresponding $C_{0}$ in return.

The figure 13 compares $\Delta G_{n^{*}}^{n u c}$ for $\mathrm{AlZr}$ at $723 \mathrm{~K}$ and $873 \mathrm{~K}$, including or not the frustration of the matrix, and the CVM solution obtained by Clouet et al. [3] for AlZr at 723K. According to this figure, the frustration effect is almost negligible at $723 \mathrm{~K}$, except at high concentration when $n^{*}$ is in the range [1-5]. The presence of cusps on the curves is due to sharp fluctuations of the free energy around values of $n$ multiple of 4 (the magnitude of the effect decreases while $n$ increases, see paper I). The CVM solution is almost parallel to the two present solutions with a shift of few meV towards higher (absolute) values of $\Delta G_{n^{*}}^{n u c}$ and a different shape when the solute concentration approaches $1 \%$; in relative values, this shift is only about $6 \%$. Neglecting the multi-exclusion reduces this shift by a factor 2 approximately. Figure 13 shows also that the influence of the frustration effect increases with temperature. Similar curves obtained for different values of $T_{w} / T$ (not reported here for sake of simplicity) show that the evolution with temperature at a given concentration is highly non linear due to the simultaneous increase of the solubility limit.

Figure 14 compares the associated critical concentrations of monomers, with and without the frustration effect. Except for very low values of $n^{*}$, for which some irregularities appear, these two curves are very similar, even if respective points corresponding to a given $n^{*}$ do not coincide. Again the frustration effect appears more pronounced at high temperature. Remark on these two figures that the range of variation of solute concentration associated with the range of variation of $n^{*}$ from 1 to 70 decreases when the temperature increases, because of the increase of the solubility limit.

\section{Figures 13-14}

To compare these results with those obtained when the frustration effect can be computed directly from KMC distributions as in section 4.2, Figure 15 compares $\Delta G_{n^{*}}^{n u c}$ as a function of the concentration of monomers. The values corresponding to KMC distributions were taken during the three precipitation kinetics reported in the previous section. As expected, the agreement between the two modes of calculation of the frustration effect is not perfect, nevertheless it is sufficient in most of the nucleation stage to validate the self consistent calculation scheme as an acceptable approximation to predict $\Delta G_{n^{*}}^{n u c}$ without performing any heavy simulations. Of course when $n^{*}$ is very small the cluster distribution used in the self-consistent mode to calculate the frustration effect represents poorly the real distributions which are much longer. Similarly when nucleation is well advanced, the real distributions are much longer than $n^{*}$. This explains why the acceptable agreement between both calculation modes concerns only the central part of the curves related to KMC simulations in Figure 15.

The evolution of $\Delta G_{n^{*}}^{n u c}(t)$ (solution "+ frustration (KMC)") during the precipitation kinetics is represented in Figure 16. The curve corresponding to the lowest supersaturated case is almost flat during the nucleation stage while for the two other cases $\Delta G_{n^{*}}^{n u c}(t)$ varies by a factor 2 from early nucleation to the end of this stage. With Figure 15, it stresses the importance to calculate the nucleation driving force consistently with the evolution of cluster concentrations as a function of the annealing time. 
Figures 15-16

\section{Conclusion}

Starting from the concept of cluster gas introduced by Frenkel, a model was developed to predict the distributions of clusters in supersaturated binary alloys. The model, which assumes that the free energy of individual clusters can be calculated with a good accuracy (cf. paper I), provides a rigorous estimation of the configuration entropy of the cluster gas, accounting for the real exclusion volume of clusters, whose values are based on a Monte Carlo calculation. A solution convenient for dilute alloys was first established then extended to account for the multi-exclusion in concentrated alloys. The predicted cluster distributions were compared with those observed in KMC simulations of $\mathrm{AlZr}$ precipitation. It was shown that when the frustration effect is neglected, the predicted distributions are never in good agreement with the $\mathrm{KMC}$ ones. A brief acceptable agreement is nevertheless observed in early nucleation but then this solution increasingly diverges with annealing time. On the contrary, accounting for the frustration effect leads to cluster distributions in excellent agreement with the KMC ones although the convergence is slower; in addition, the agreement persists approximately until the number of stable clusters decreases. The correction of absorption / emission coefficients in cluster dynamics was established independently of the scheme chosen to solve the master equation. Finally the results were examined at the light of the classical theory of nucleation, revisiting some usual approximations. It was shown that whatever the calculation method adopted to predict the precipitation kinetics, the whole cluster distribution needs to be accounted for to correctly predict the nucleation free energy. However, in dilute alloys, this calculation can be restricted to subcritical clusters as usually done in simple thermodynamical models. This model can be applied to any binary alloys, provided that clusters can be considered as non-interacting, but requires various quantities like the cluster free energy to be calculated accurately as a function of the ratio $T_{w} / T$ for given structures of clusters and matrix. The obtained results can be used to evaluate the degree of approximation of various general models and calculation methods like CVM, for instance. Finally, the best way to conclude this work is certainly to compare cluster distributions obtained in $\mathrm{CD}$ with and without this frustration effect taken into account, with $\mathrm{KMC}$ results for $\mathrm{AlZr} 3 \%$ at $873 \mathrm{~K}$, as reported in figure 17. The most simple scheme of $\mathrm{CD}$ was used, so that with help of fitting data provided in paper I for cluster free energy $\left(T_{w} / T \approx 1\right)$ and present table 1 , reproducing present results should not present any major difficulties (see [17] for basic equations). The effect is so important at this temperature that one cannot expect correct predictions for the kinetics if it is not accounted for. Of course, for these low solubility alloys, when the temperature decreases the effect decreases quickly, but inversely, for alloys of higher solubility, like AlLi for instance, this effect should be much more severe. If extending the calculation of cluster free energy as done in paper I to multicomponent alloys is a challenge, on the contrary, the present frustration effect is not difficult to generalize; $\mathrm{AlZrSc}$ alloys could be good candidates for such an application. Finally, it is hoped that this work has successfully demonstrated that we still have to learn from ordinary binary alloys.

\section{Figures 17}

\section{Acknowledgments}

This work greatly benefited from the joint research program "CPR Precipitation" (Arcelor, Pechiney, CNRS and CEA). Various discussions with Drs. G. Martin, F. Soisson, A. Finel and P. Maugis are gratefully acknowledged. The author is especially grateful to Dr. E. Clouet for stimulating discussions and for providing his KMC data. 


\section{References}

[1] SCHMELZER, J., (Ed.), 2005, "Nucleation Theory and Applications", Wiley-VCH, Weinheim.

[2] BELLON, P., 2003, in "Thermodynamics, Microstructures and Plasticity", Eds. A. Finel, D. Maziere and M. Veron, Kluwer Academic Publisher, Dordrecht, The Netherlands.

[3] ClOUET, E., NASTAR, M. and SIGLI, C., 2004, Phys. Rev. B, 69, 064109.

[4] SOISSON, F. and MARTIN, G., 2000, Phys. Rev. B, 62, 203.

[5] KELTON, K., 2000, Acta Mater., 48, 1967

[6] LEPINOUX, J., 2005, Philosophical Magazine, 85, 3585

[7] SLESOV, V. and SCHMELZER, J., 1994, J.Chem. Solids, 55, 243.

[8] MARTIN, G., 2006, to be appear in Mat. Scie. Eng.

[9] PERRINI, A., JACUCCI, G. and MARTIN, G., 1984a, Surface Science, 144, 53.

[10] NARSIMHAN, G. and RUCKENSTEIN, E., 1989, J. Colloid Interface Sci., 137, 583.

[11] NOWAKOWSKI, B. and RUCKENSTEIN, E., 1991, J. Chem. Phys., 94, 1397

[12] FRENKEL, J., 1955, Kinetic Theory of Liquids, New York, Dover Publications.

[13] FINEL,A., 1994, in Statics and Dynamics of Phase Transformations (P. Turchi and A. Gonis, Eds.), Plenum Press, New York, p.495-540

[14] DUCASTELlE, F., 1991, Order and Phase Stability in Alloys, North-Holland, Amsterdam.

[15] DOMB, C., 1974, in "Phase Transformations and Critical Phenomena", (C. Domb and M.S. Green, Eds), Academic, London, p. 357.

[16] CLOUET, E. 2004, PhD thesis, Ecole Centrale, Paris. Available online at: tel.csd.cnrs.fr/documents/archives0/00/00/59/74

[17] CLOUET, E., BARBU, A., LAE, L. and MARTIN, G., 2005, Acta Materiala., 53, 2343

[18] LAE, L., GUYOT, P. and SIGLI, C., 2005, in Proceedings of the $9^{\text {th }}$ ICAA (J. Nie, A. Morton, B. Muddle, Eds.), Institute of Materials Engineering Australia. Ltd., p.281-286.

[19] KASHCHIEV, D., 1969, Surface Sci., 14, 209.

[20] WAGNER, R. and KAMPMANN, R, 1991, in "Phase Transformations in Materials", Materials Science and Technology (R.W. Cahn, P. Haasen and E.J. Krame, Eds.), Vol. 5, VCH Weinheim, p. 215-303.

[21] MAUGIS, P. and GOUNE, M., 2005, Acta Materiala, 53, 3359. 


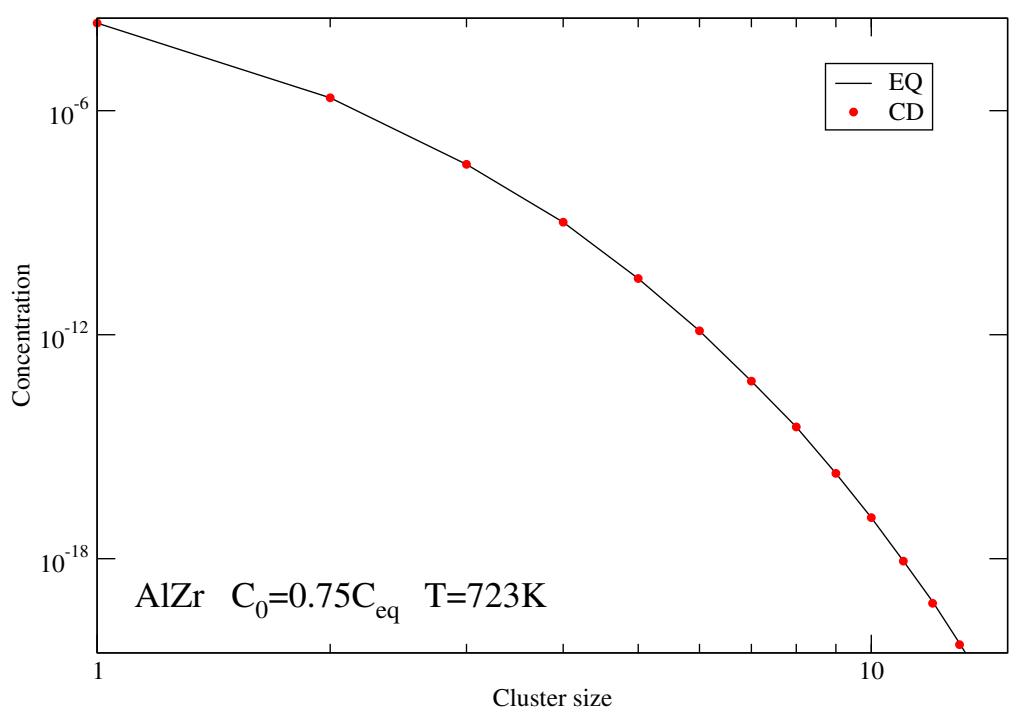

Figure 1: Equilibrium distribution of clusters obtained in an undersaturated alloy of low solubility limit. EQ: from Equation (2); CD: from classical cluster dynamics.

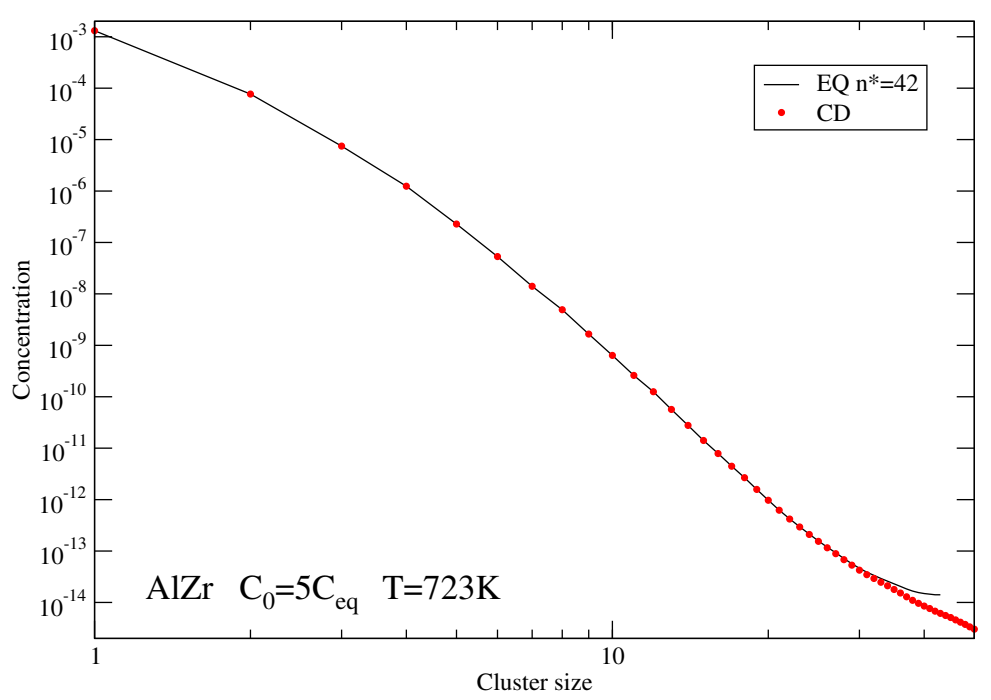

Figure 2: Equilibrium distribution of clusters obtained in a low supersaturated alloy of low solubility limit. EQ: from Equation (2) plotted up to the critical cluster size $n^{*}=42$; CD: from classical cluster dynamics. 
Figure 3: Exclusion volume $V_{l n}$ of monomers by $n$-mers (or inversely), normalized by $n$. The bottom of the figure represents the asymptotic limit for infinite $n$ (equal to 4 for $\mathrm{L}_{12}$ clusters). For AlZr alloys, $T_{w} / T=1.350$ and 1.072 correspond to $723 \mathrm{~K}$ and $873 \mathrm{~K}$, respectively. $T_{w} / T=0.875$ and 0.750 correspond to intermediate cases between low solubility limit alloys like AlZr or AlSc, and high solubility limit alloys like AlLi, for instance.

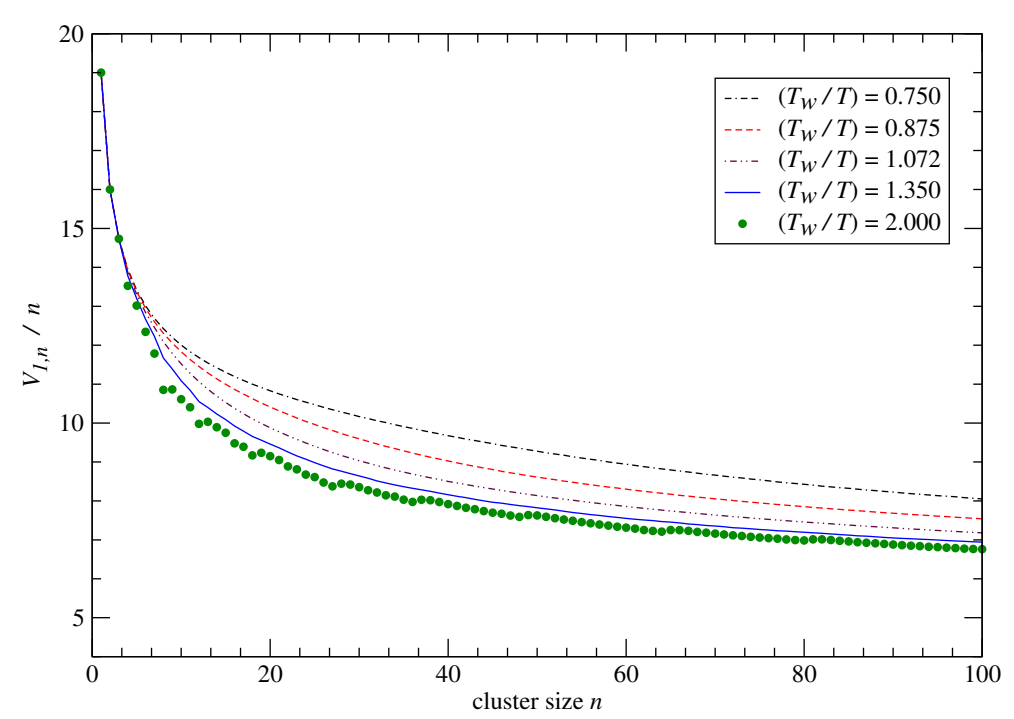

Figure 4: Exclusion volumes of clusters of size $\mathrm{J}$ by clusters of size I, and inversely. For this evaluation, all clusters are taken spherical. 
Figure 5: Exclusion radius $R_{n}$ normalized by the radius of a sphere of volume equal to $n$ times the unit cell $a^{3}$. The asymptotic limit for infinite $n$ is equal to 1 .

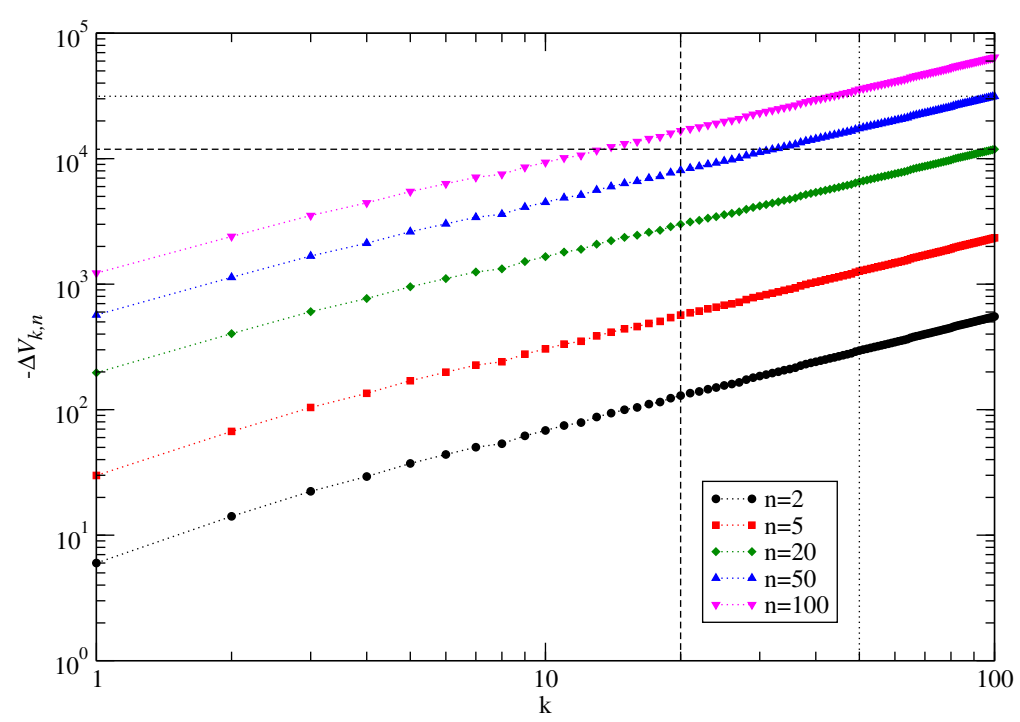

Figure 6: Variation of the frustration factor $-\Delta V_{k, n}$ with $k$ for a few values of $n$. Results are almost independent on $T_{n} / T$. The two pairs of dashed and dotted lines allow for comparing the evolution of $\Delta V_{k, n} v s . \Delta V_{n, k}$ for $n=100$, $k=20$ and 50. 


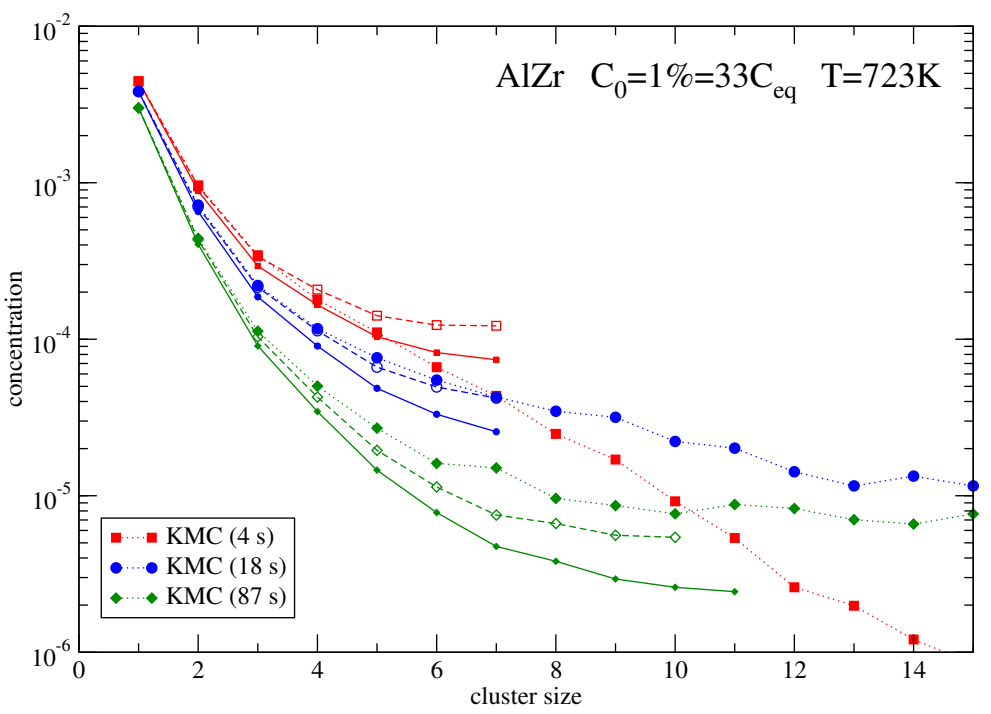

Figure 7: Compa temperature and represented by di set of 3 curves co simulations of AlZr at medium with and without frustration are rely, for 3 different times. Each

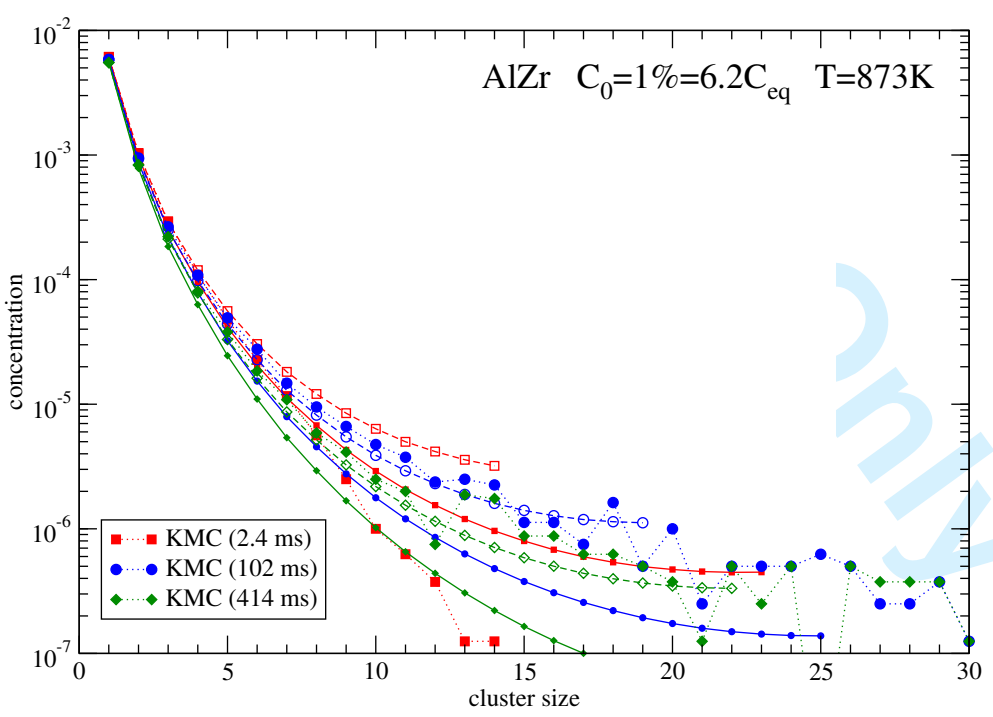

Figure 8: Comparison of predicted distributions with those obtained by KMC simulations of AlZr at high temperature and medium concentration (by courtesy from E. Clouet). Solutions with and without frustration are represented by dashed and plain lines, empty and small filled symbols, respectively, for 3 different times. Each set of 3 curves corresponds to a particular concentration of monomers. Symbols near the bottom of the figure correspond to single clusters in the corresponding classes of size. 
Figure 9: Comparison of predicted distributions with those obtained by KMC simulations of $\mathrm{AlZr}$ at high temperature and high concentration (by courtesy from E. Clouet). Solutions with and without frustration are represented by dashed and plain lines, empty and small filled symbols, respectively, for 3 different times. Each set of 3 curves corresponds to a particular concentration of monomers.

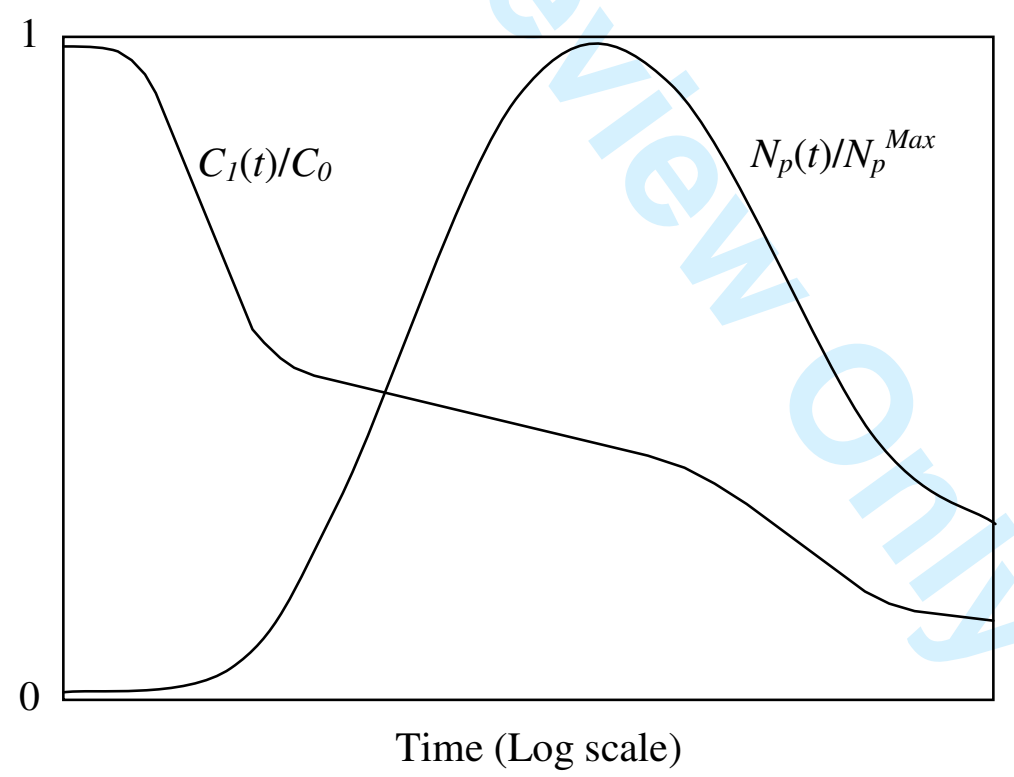

Figure 10: Schematic representation of the evolution of both the concentration of monomers $C_{l}(t)$ normalized by the total solute concentration $C_{0}$, and the number of precipitates $N_{p}(t)$ (of size greater than a critical size) normalized by its maximum value. The respective positions, lengths and variations of slope of the different stages may significantly vary with $C_{0}$ and $T_{w} / T$. 


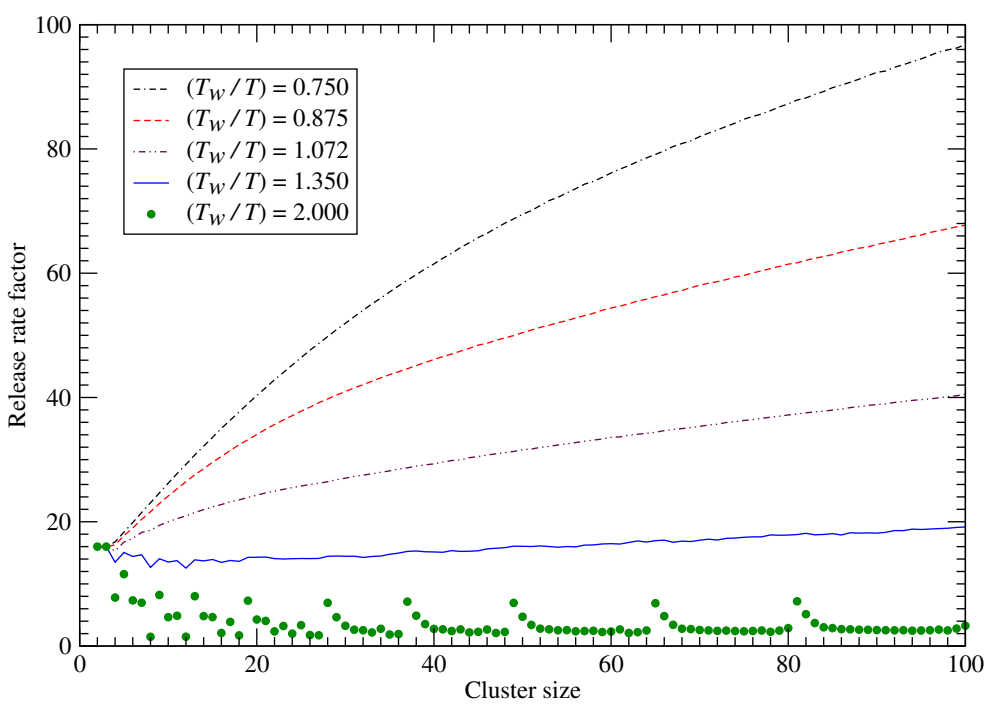

Figure 11: Release rate factor $\bar{P}_{n, n+1}^{0}$ (Eq. 31) for the $\mathrm{L}_{12}$ structure normalised by $\vee \operatorname{Exp}\left(-T_{w} / T\right) ; T_{w} / T=1.35$ and 1.07 correspond to $\mathrm{T}=723 \mathrm{~K}$ and $873 \mathrm{~K}$ respectively.

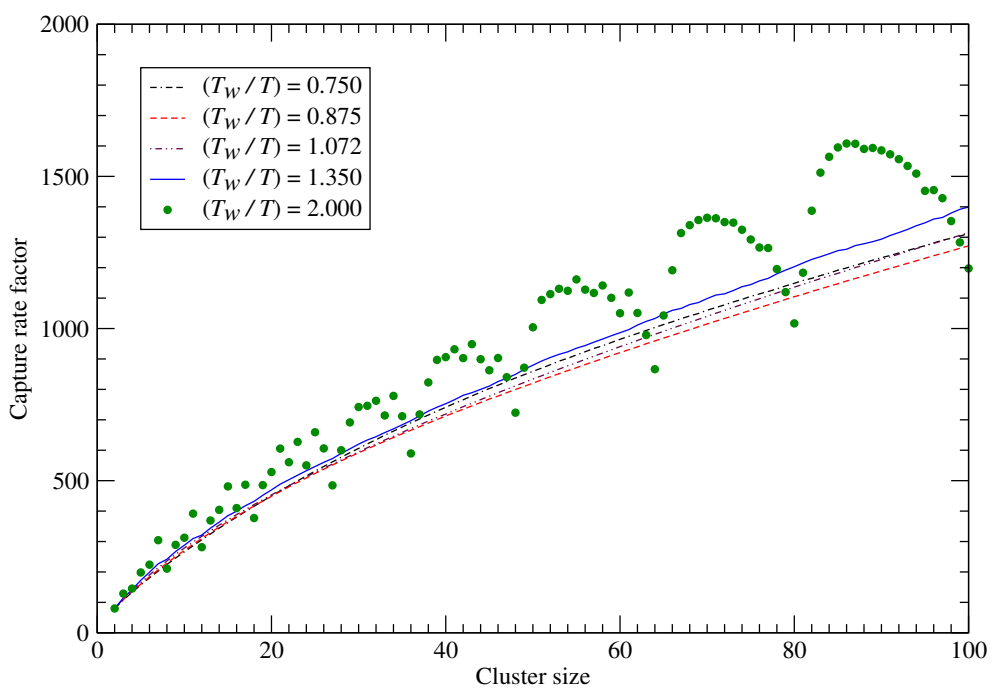

Figure 12: Capture rate factor $\bar{P}_{n, n+1}^{0}$ (Eq. 32) for $\mathrm{L}_{12}$ structure normalised by $\vee \operatorname{Exp}\left(T_{w} / T\right) ; T_{w} / T=1.35$ and 1.07 correspond to $\mathrm{T}=723 \mathrm{~K}$ and $873 \mathrm{~K}$ respectively. 
Figure 13: Comparison of the predicted values of the nucleation free energy with $(+)$ and without (-) frustration (from the right to the left $n^{*}$ varies from 1 to 70) with the CVM calculation performed by Clouet et al., 2004

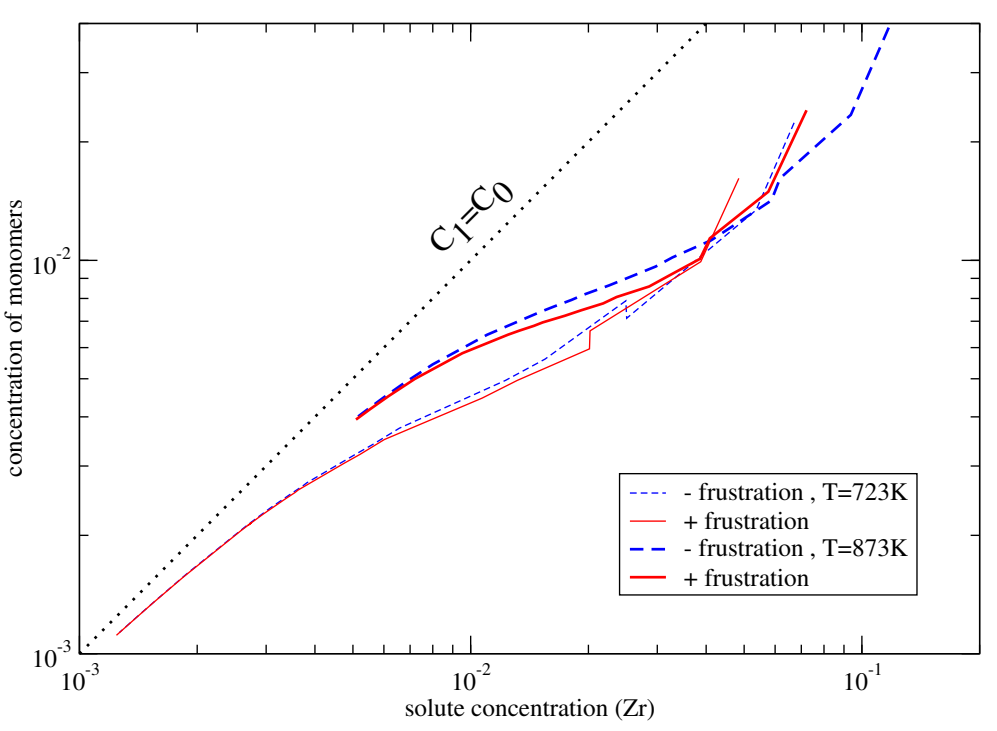

Figure 14: Evolution of the concentration of monomers associated with figure 13. The straight dotted line represents a distribution containing only monomers. 


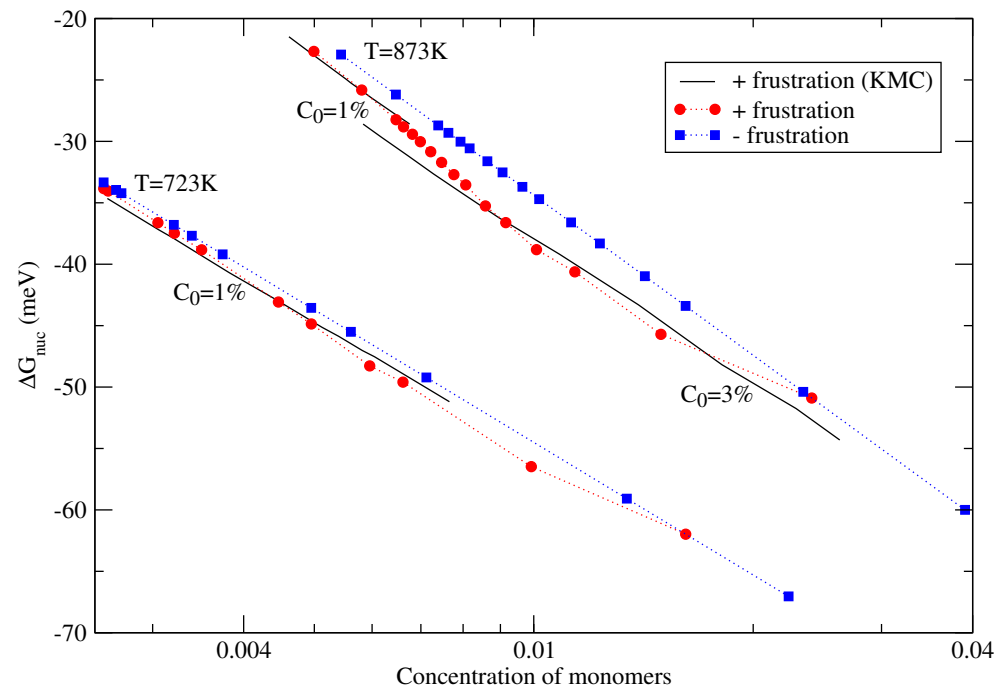

Figure 15: Comparison of the two calculation modes of the nucleation free energy. The symbols are relative to the simplest mode with (+ frustration) and without (- frustration) frustration reported in figures 13-14. For curves ("+ frustration (KMC)") the frustration effect is calculated from KMC distributions relative to the same kinetics than in figures (6-9).

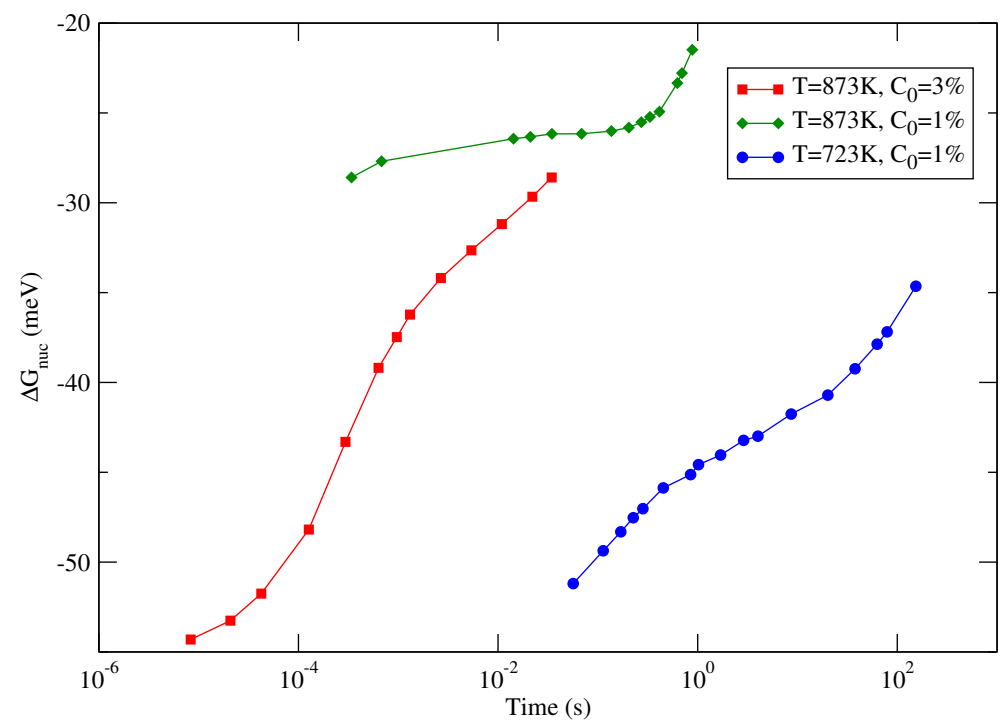

Figure 16: Evolution of the nucleation free energy (solution "+ frustration (KMC)" in figure 15) during KMC precipitation kinetics used in Figures (7-9). 
Figure 17: Comparison of 2 KMC cluster distributions (by courtesy from E. Clouet) with those obtained in CD, with and without matrix frustration accounted for, for the same concentrations in monomers. Note that the smallest possible cluster concentration for these KMC results was $10^{-6}$, i.e. one cluster for $10^{6}$ atomic sites.

\begin{tabular}{|c|c|c|c|}
\hline Tw/T & $\mathbf{a}$ & $\mathbf{b}$ & $\mathbf{c}$ \\
\hline 1.350 & 8.580 & 11.885 & -2.229 \\
\hline 1.072 & 10.554 & 12.682 & -4.230 \\
\hline 0.875 & 13.995 & 12.820 & -5.731 \\
\hline
\end{tabular}

Table 1: Fit of $V_{1, n}$ with expression: $V_{1, n}=4 n+a n^{2 / 3}+b n^{1 / 3}+c$ 
$\underline{\text { Table and Figures captions }}$

Table 1: Fit of $V_{1, n}$ with expression: $V_{1, n}=4 n+a n^{2 / 3}+b n^{1 / 3}+c$

Figure 1: Equilibrium distribution of clusters obtained in an undersaturated alloy of low solubility limit. EQ: from Equation (2); CD: from classical cluster dynamics.

Figure 2: Equilibrium distribution of clusters obtained in a low supersaturated alloy of low solubility limit. EQ: from Equation (2) plotted up to the critical cluster size $n^{*}=42$; CD: from classical cluster dynamics.

Figure 3: Exclusion volume $V_{1, n}$ of monomers by $n$-mers (or inversely), normalized by $n$. The bottom of the figure represents the asymptotic limit for infinite $n$ (equal to 4 for $\mathrm{L}_{12}$ clusters). For AlZr alloys, $T_{w} / T=1.350$ and 1.072 correspond to $723 \mathrm{~K}$ and $873 \mathrm{~K}$, respectively. $T_{w} / T=0.875$ and 0.750 correspond to intermediate cases between low solubility limit alloys like AlZr or AlSc, and high solubility limit alloys like AlLi, for instance.

Figure 4: Exclusion volumes of clusters of size $\mathrm{J}$ by clusters of size I, and inversely. For this evaluation, all clusters are taken spherical.

Figure 5: Exclusion radius $R_{n}$ normalized by the radius of a sphere of volume equal to $n$ times the unit cell $a^{3}$. The asymptotic limit for infinite $n$ is equal to 1 .

Figure 6: Variation of the frustration factor $-\Delta V_{k, n}$ with $k$ for a few values of $n$. Results are almost independent on $T_{w} / T$. The two pairs of dashed and dotted lines allow for comparing the evolution of $\Delta V_{k, n}$ vs. $\Delta V_{n, k}$ for $n=100, k=20$ and 50 .

Figure 7: Comparison of predicted distributions with those obtained by KMC simulations of $\mathrm{AlZr}$ at medium temperature and medium concentration (by courtesy from E. Clouet). Solutions with and without frustration are represented by dashed and plain lines, empty and small filled symbols, respectively, for 3 different times. Each set of 3 curves corresponds to a particular concentration of monomers.

Figure 8: Comparison of predicted distributions with those obtained by KMC simulations of $\mathrm{AlZr}$ at high temperature and medium concentration (by courtesy from E. Clouet). Solutions with and without frustration are represented by dashed and plain lines, empty and small filled symbols, respectively, for 3 different times. Each set of 3 curves corresponds to a particular concentration of monomers. Symbols near the bottom of the figure correspond to single clusters in the corresponding classes of size. 
Figure 9: Comparison of predicted distributions with those obtained by KMC simulations of $\mathrm{AlZr}$ at high temperature and high concentration (by courtesy from E. Clouet). Solutions with and without frustration are represented by dashed and plain lines, empty and small filled symbols, respectively, for 3 different times. Each set of 3 curves corresponds to a particular concentration of monomers.

Figure 10: Schematic representation of the evolution of both the concentration of monomers $C_{1}(t)$ normalized by the total solute concentration $C_{0}$, and the number of precipitates $N_{p}(t)$ (of size greater than a critical size) normalized by its maximum value. The respective positions, lengths and variations of slope of the different stages may significantly vary with $C_{0}$ and $T_{w} / T$.

Figure 11: Release rate factor $\bar{P}_{n, n+1}^{0}$ (Eq. 31) for the $\mathrm{L}_{12}$ structure normalised by $\vee \operatorname{Exp}\left(-T_{w} / T\right)$; $T_{n} / T=1.35$ and 1.07 correspond to $\mathrm{T}=723 \mathrm{~K}$ and $873 \mathrm{~K}$ respectively.

Figure 12: Capture rate factor $\bar{P}_{n, n+1}^{0}$ (Eq. 32) for $\mathrm{L}_{12}$ structure normalised by $\vee \operatorname{Exp}\left(T_{w} / T\right)$; $T_{w} / T=1.35$ and 1.07 correspond to $\mathrm{T}=723 \mathrm{~K}$ and $873 \mathrm{~K}$ respectively.

Figure 13: Comparison of the predicted values of the nucleation free energy with $(+)$ and without (-) frustration (from the right to the left $n^{*}$ varies from 1 to 70 ), with the CVM calculation performed by Clouet et al., 2004

Figure 14: Evolution of the concentration of monomers associated with figure 13. The straight dotted line represents a distribution containing only monomers.

Figure 15: Comparison of the two calculation modes of the nucleation free energy. The symbols are relative to the simplest mode with ("+ frustration") and without ("- frustration") frustration reported in figures 13-14. For curves ("+ frustration (KMC)") the frustration effect is calculated from KMC distributions relative to the same kinetics than in figures (6-9).

Figure 16: Evolution of the nucleation free energy (solution "+ frustration (KMC)" in figure 15) during KMC precipitation kinetics used in Figures (7-9).

Figure 17: Comparison of 2 KMC cluster distributions (by courtesy from E. Clouet) with those obtained in $\mathrm{CD}$, with and without matrix frustration accounted for, for the same concentrations in monomers. Note that the smallest possible cluster concentration for these $\mathrm{KMC}$ results was $10^{-6}$, i.e. one cluster for $10^{6}$ atomic sites. 